\section{Case Reports in Oncology}

\title{
Mesothelioma: A Case in a Diagnostic Timeline and the Efficiency of Robot-Assisted Surgery
}

\author{
Paul Zarogoulidis ${ }^{a}$ b Kosmas Tsakiridis $^{b}$ Thomas Zarampoukas ${ }^{c}$ \\ Katerina Zarampouka ${ }^{c}$ Dimitris Hatzibougias ${ }^{d}$ Christos Tolis $^{\mathrm{e}}$ \\ Stavros Tryfon ${ }^{f}$ Maria Saroglou $^{f}$ Aris loannidisg ${ }^{\text {Dimitris Matthaios }}{ }^{\mathrm{h}}$ \\ Christoforos Kosmidis ${ }^{\text {a }}$

\footnotetext{
a3rd Surgery Department, "AHEPA" University General Hospital, Thessaloniki, Greece; bPulmonary Department, "General Clinic" Euromedica, Thessaloniki, Greece; 'Private

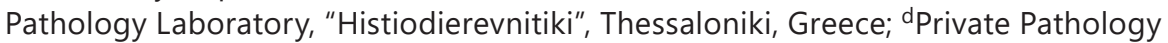
Laboratory, "Microdiagnostics", Thessaloniki, Greece; eprivate Oncology Laboratory, "Oncoderm", Ioannina, Greece; ${ }^{\prime}$ Pulmonary Department, "G. Papanikolaou" General Hospital, Thessaloniki, Greece; 'S Surgery Department, "Genesis" Private Hospital, Thessaloniki, Greece; ' Oncology Department, General Hospital of Rhodes, Rhodes, Greece
}

\section{Keywords}

Mesothelioma · Video-assisted thoracic surgery · CT-guided biopsy · Biopsy · Thorax · Atypia

\begin{abstract}
We present a case of a 65 -year-old male with a difficulty to diagnose mesothelioma. To be specific, three attempts were made to diagnose the disease, and only with a large sample performed with robot-assisted surgery, our pathologists were able to identify the malignancy. The novelty for our case is mostly based on the timeline of the diagnosis along with the tissue samples where we present the course of the transformation from benign to malignancy. All tissue biopsies were checked by two independent pathologists. Conclusively, diagnosis for small local lesions should be performed with an endoscopic method, video-assisted or robot-assisted.
\end{abstract}

\section{Karger $\stackrel{2}{\circ}$}




\section{Introduction}

Mesothelioma is known to be associated to asbestos exposure, it does not have early disease signs, and therefore, it is usually diagnosed at the advanced unoperable disease stage. Usually, most patients are admitted to a hospital with shortness of breath which started several months before, and a radiology examination reveals pleura effusion and pleura thickness. As the first step, we usually drain the pleura effusion by placing a chest tube, and a biopsy is performed usually with three ways: (a) transthoracic ultrasound with a convex probe - 18G needle, (b) computed tomography guided biopsy - 18G needle, and (c) videoassisted thoracic surgery or even robot-assisted thoracic surgery with large tissue sample [1]. Then, staging is performed. Pleura effusion can be used for diagnosis by using the cell block methodology [2]. Unfortunately, most patients are diagnosed at a late stage, and systemic treatment is initiated. Novel biomarkers are being investigated for early disease diagnosis [3]. We present a case of mesothelioma in a diagnostic timeline.

\section{Case Report}

We report a case of a 65-year-old male with known exposure to asbsestos. He was admitted to our outpatient ward for the investigation of persistent cough ( 6 months) and mild back pain on the lower right hemithorax. A computed tomography of the thorax without intravenous (i.v.) contrast revealed a pleural thickness of $1 \mathrm{~cm}$. A biopsy was performed with a eub-6500HV ultrasound source and convex probe (Fig. 1). The first pathology report did not reveal cancer (Fig. 2). It was proposed that the findings were due to an old infection, and paracetamol and codeine oral suspension were administered. In the upcoming follow-up after 1 year with a computed tomography without i.v., the pleural thickness was increased to $1.7 \mathrm{~mm}$ (width), and again, another biopsy under computed tomography was performed with a 18G needle, only this time the pathology report revealed atypia, a precancerous condition (Fig. 3). However, no treatment was provided, and only follow-up was proposed. In the next 8 months, a new computed tomography was performed without i.v., and the lesion had clearly
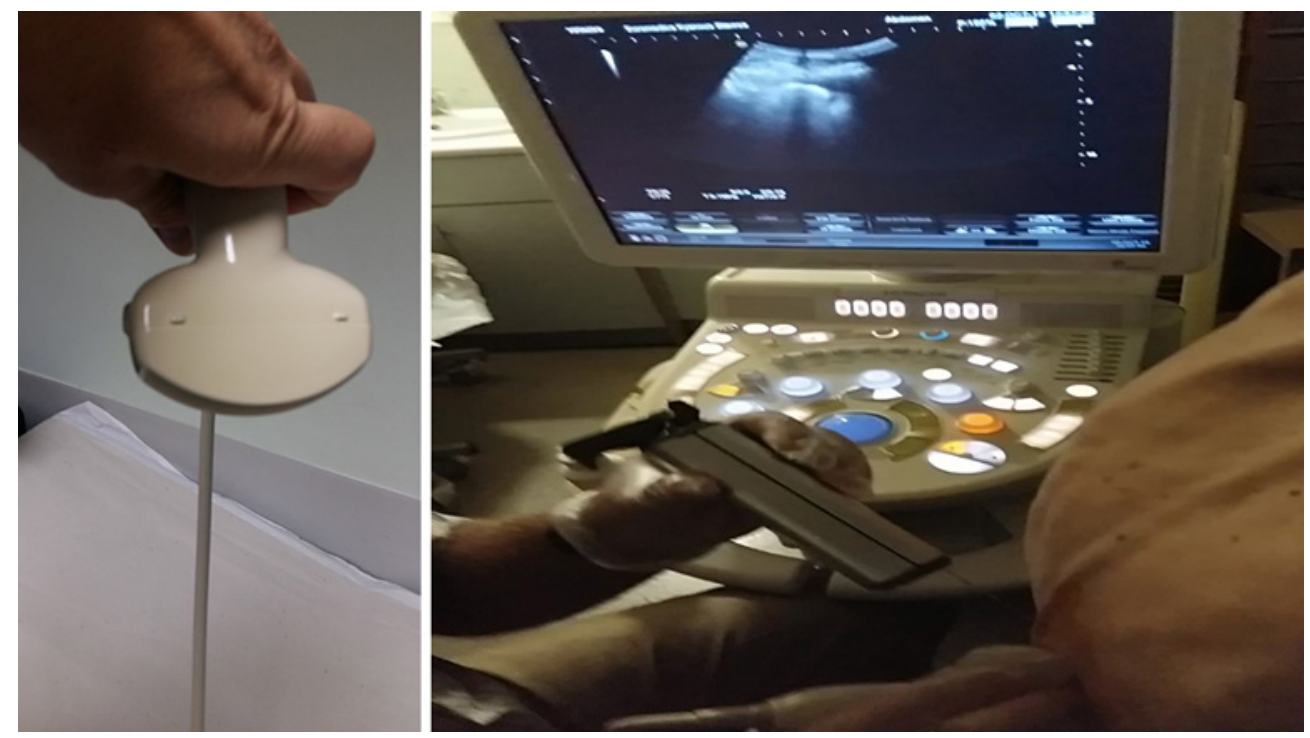

Fig. 1. Left; the convex probe, right; during the first biopsy.

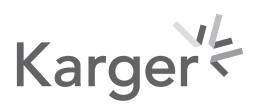




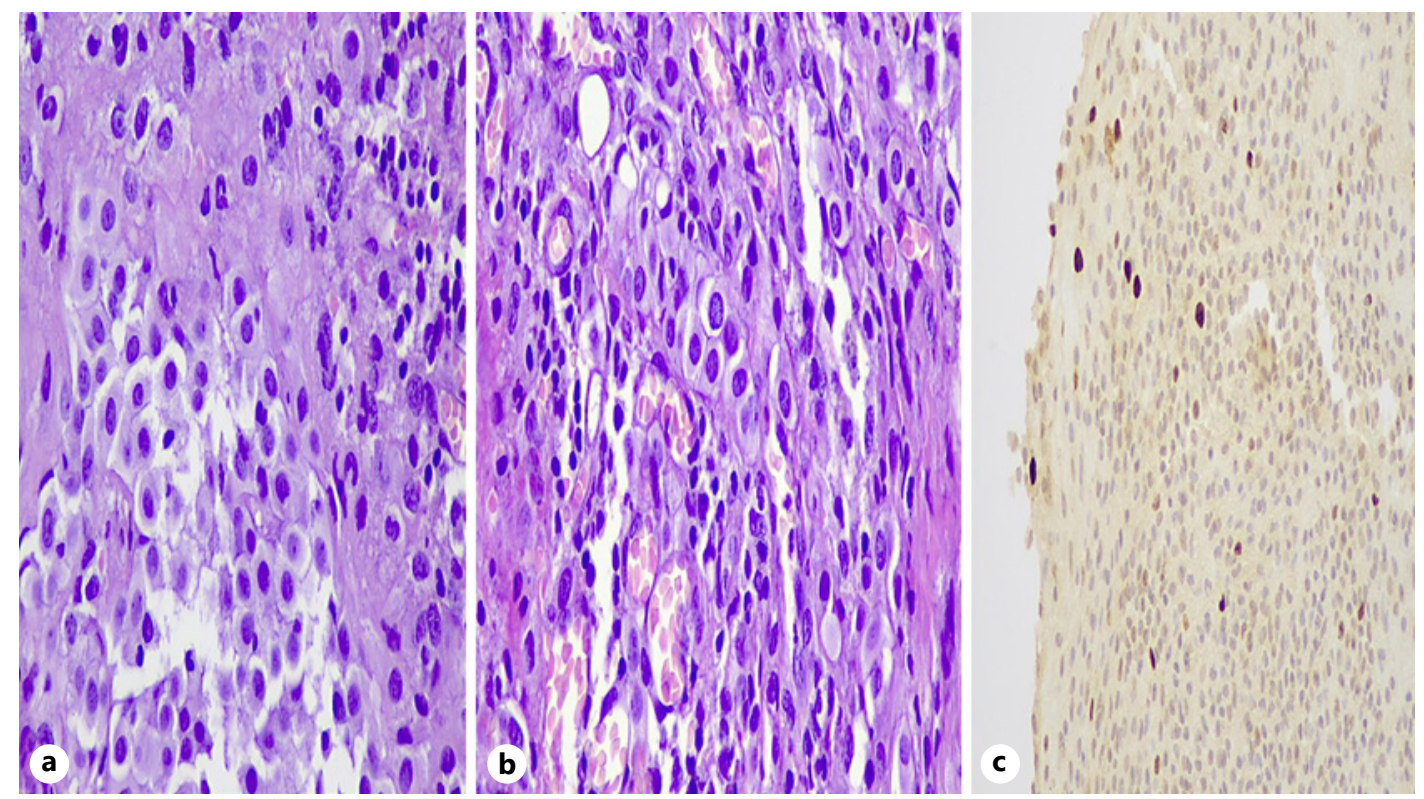

Fig. 2. a-b M28186_HE $\times 400$ and M28186_HE $\times 400 \_2$ : hyperplastic mesothelial cells without atypia, in a hemorrhagic, fibrous, and hyalinized stroma (H\&E ×400). c M28186_Ki67 ×400_3: Ki67 proliferation index was less than $5 \%$.
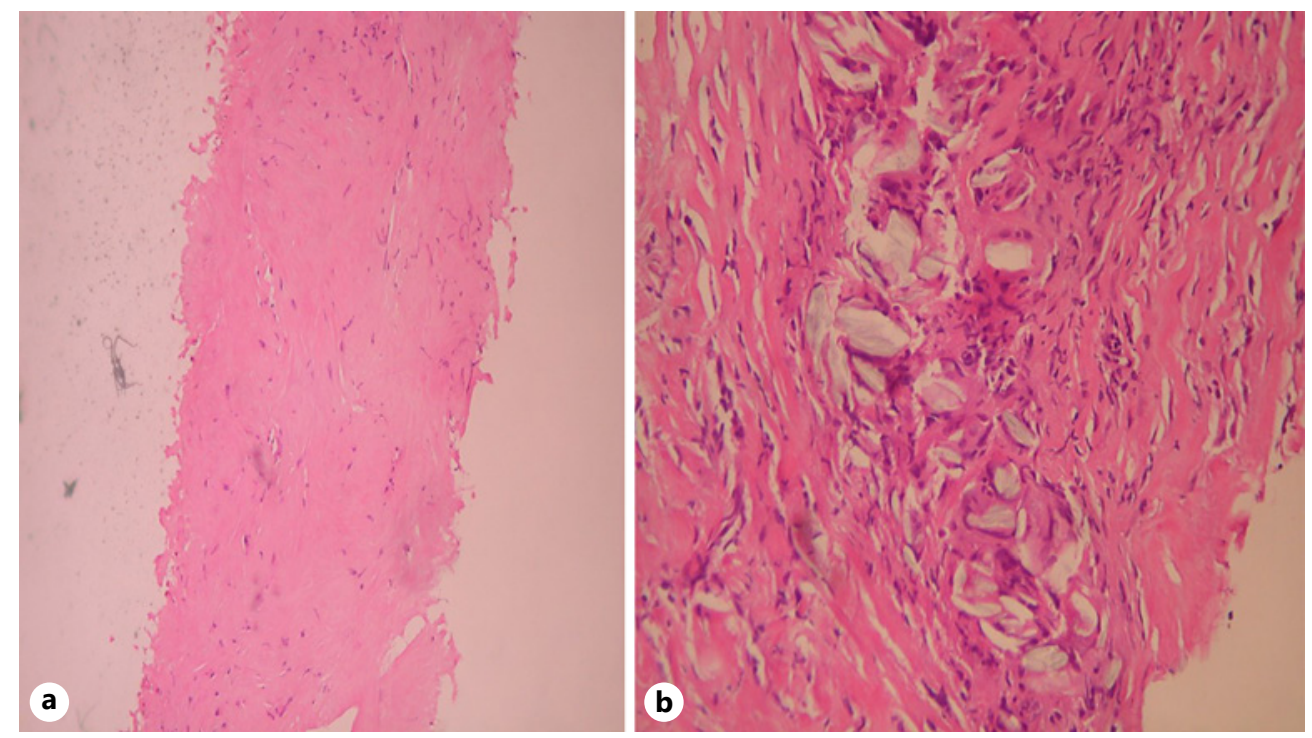

Fig. 3. a Dense collagenous fibrous tissue $(H \& E \times 100)$. b Foreign body giant-cell reaction $(H \& E \times 200)$.

increased to $2.5 \mathrm{~cm}$ (width), and video-assisted thoracic surgery was performed, and mesothelioma was revealed (Fig. 4). The neoplasm consisted of spindle and few epithelioid cells without significant nuclear atypia or mitosis, in dense fibrotic stroma. Immunohistochemistry showed that the tumor cells were positive for vimentin and CKAE1/AE3 and showed limited positivity for WT1, D2-40, EMA, and calretinin and were negative for CK5/6. The nuclear positivity for Ki-67 was about $20 \%$. From the above findings, the diagnosis of mesothelioma, probably desmoplastic type, was confirmed (Fig. 5-6). Pleurodesis was performed during the diagnostic procedure. 

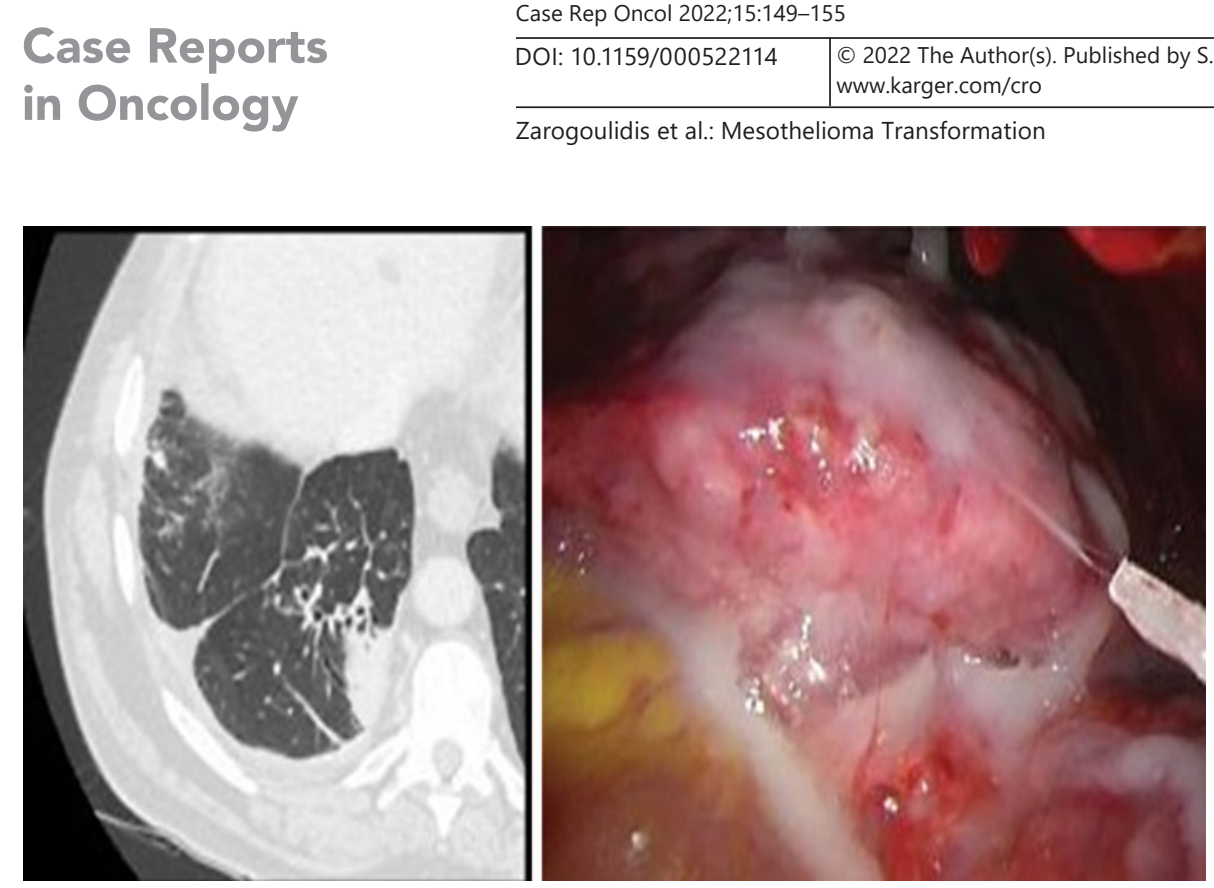

Fig. 4. Left: computed tomography with the lesion and right: the mass during the RATS and pleurodesis. RATS, robot-assisted thoracic surgery.
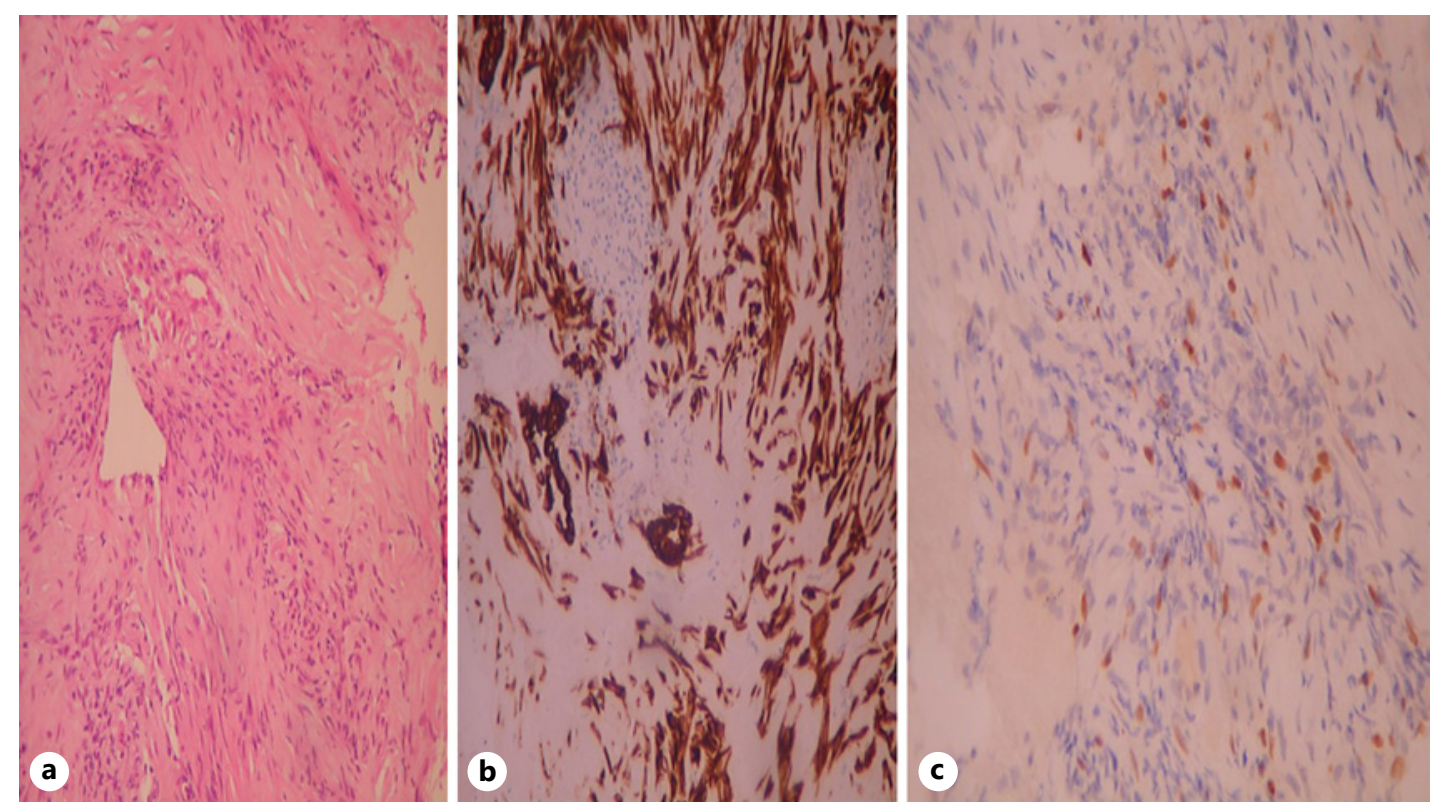

Fig. 5. a Spindle and few epithelioid cells in fibrotic stroma $(H \& E \times 100)$. b Tumor cells are positive for CKAE1/AE3 (immunostain $\times 200$ ). c Tumor cells show limited positivity for WT1 (immunostain $\times 200$ ).

\section{Discussion}

There are different types of mesothelioma: (a) pleural, peritoneal, pericardial, and testicular. In case of early disease, several types of surgeries can be performed, such as, extrapleural pneumonectomy, where we remove the affected lung along with chest lymph nodes, heart lining, and diaphragm and pleurectomy decortication, where the lung is spared, and we remove only the affected pleura lining, chest wall, heart lining, and diaphragm. It is considered a less invasive type of surgery [4]. In general, both surgeries are considered extremely amputative, and in some cases, additional chemotherapy with or without radiotherapy is necessary. 

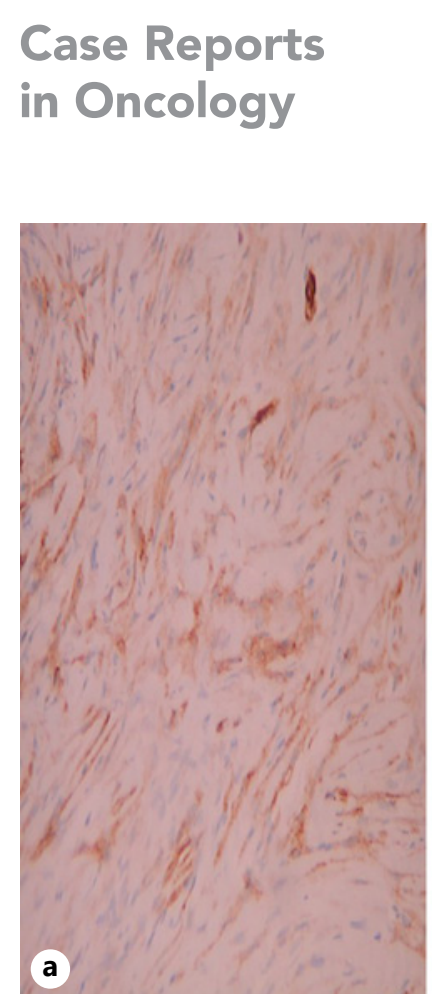

Fig. 6. a Limited positivity for $D 2-40$ (immunostain $\times 200$ ). b Limited positivity for EMA (immunostain $\times 200$ ). c Enough cells show nuclear positivity for Ki-67 (immunostain $\times 200$ ).

Surgery severely reduces the quality of life of a patient, and rehabilitation is needed. In the case of inoperable disease, first-line chemotherapy treatment includes cisplatin with pemetrexed, and as second-line chemotherapy, it may include carboplatin with Gemzar [5]. Other chemotherapy drugs and combinations have been also used [6, 7]. There are also other protocols as second- of third-line treatment that include immunotherapy and local treatment [8]. Several different treatments have been investigated like gene therapy, cryotherapy ablation, photodynamic therapy, virotherapy, and hyperthermia intrathoracic chemotherapy which is experimental $[5,9,10]$. Radiation such as external-beam radiation therapy can be used before or during surgery. Moreover, brachytherapy can be performed by placing radioactive implants inside the lesion, and it can be combined with other conventional treatments. Staging for mesothelioma includes computed tomography and magnetic reconnaissance and bone scintigraphy. Positron emission tomography is currently being investigated whether it could be used for mesothelioma along with convex EBUS-TBNA and EUS-TBNA [9, 11-14]. In the case where treatment is not efficient and the pleura effusion is increased, thoracentesis by putting a needle in the affected lung provides immediate relief, or a PleurXcatheter is placed as a permanent catheter in the thoracic cavity to drain the pleural fluid. Another method is pleurodesis where we apply talc poudrage inside the affected hemithorax as a method for stopping the pleural effusion production. At the same time, we can administer local radiotherapy. In the case of ascites, we can perform paracentesis with a needle or a catheter under the guidance of convex probe ultrasound. In the case of pericardial fluid, we can perform pericardiocentesis. We should never forget that we need a balanced diet rich in protein and energy for all these patients [15].

\section{Conclusion}

In our case, our patient had bone metastasis, and chemotherapy was initiated along with radiotherapy. Our patient after 6 months has a good quality of life and is under treatment. 


\section{Case Reports in Oncology}

\begin{tabular}{l|l}
\hline Case Rep Oncol 2022;15:149-155 \\
\hline DOI: 10.1159/000522114 & $\begin{array}{l}\text { @ 2022 The Author(s). Published by S. Karger AG, Basel } \\
\text { www.karger.com/cro }\end{array}$ \\
\hline
\end{tabular}

Zarogoulidis et al.: Mesothelioma Transformation

\section{Statement of Ethics}

Written informed consent was obtained from the patient for publication of the details of their medical case and any accompanying images. The investigational review board of Intebalkan European Medical Center of the Thoracic Surgery Department approved the case with the identification number $1 / 21$.

\section{Conflict of Interest Statement}

The authors have no conflicts of interest to declare.

\section{Funding Sources}

No funding was received.

\section{Author Contributions}

P.Z., K.T., T.Z., K.Z., D.C., C.T., S.T., M.S., A.I., D.M., and C.K. collected the information and wrote the manuscript.

\section{Data Availability Statement}

All data can be provided if requested from Dr. Paul Zarogoulidis (email: pzarog@hotmail. com) and Dr. Kosmas Tsakiridis (email: kosjohn@otenet.gr). Data can be found in the archive of the European Interbalkan Medical Center, Thessaloniki, Greece, if requested. Data are not publicly available by the Personal Information Protection ACT of Greece.

\section{References}

1 Barbieri PG, Calisti R, Calabresi C. Pleural malignant mesotheliomas from environmental exposures to asbestos in Italy. Epidemiol Prev. 2021 Jul-Aug;45(4):289-95.

2 Monaco SE, Brcic L, Dacic S. State-of-the-art cytology of pleural fluid, focusing on the diagnosis of mesothelioma. Cytopathology. 2022 Jan;33(1):57-64.

3 Girolami I, Lucenteforte E, Eccher A, Marletta S, Brunelli M, Graziano P, et al. Evidence-based diagnostic performance of novel biomarkers for the diagnosis of malignant mesothelioma in effusion cytology. Cancer Cytopathol. 2021 Sep 3. Epub ahead of print.

4 Foroulis CN, Kleontas AD, Tagarakis G, Nana C, Alexiou I, Grosomanidis V, et al. Massive chest wall resection and reconstruction for malignant disease. Onco Targets Ther. 2016;9:2349-58.

5 Porpodis K, Zarogoulidis P, Boutsikou E, Papaioannou A, Machairiotis N, Tsakiridis K, et al. Malignant pleural mesothelioma: current and future perspectives. J Thorac Dis. 2013 Sep;5(Suppl 4):S397-406.

6 Zarogoulidis P, Mavroudi M, Porpodis K, Domvri K, Sakkas A, Machairiotis N, et al. Pegylated liposomal doxorubicin in malignant pleural mesothelioma: a possible guardian for long-term survival. Onco Targets Ther. 2012;5:231-6.

7 Romero D. Benefit with ramucirumab in mesothelioma. Nat Rev Clin Oncol. 2021 Nov;18(11):676.

8 Nakajima EC, Vellanki PJ, Larkins E, Chatterjee S, Mishra-Kalyani PS, Bi Y, et al. FDA approval summary: nivolumab in combination with ipilimumab for the treatment of unresectable malignant pleural mesothelioma. Clin Cancer Res. 2021 Aug 30. Epub ahead of print.

9 Aprile V, Lenzini A, Lococo F, Bacchin D, Korasidis S, Mastromarino MG, et al. Hyperthermic intrathoracic chemotherapy for malignant pleural mesothelioma: the forefront of surgery-based multimodality treatment. J Clin Med. 2021 Aug 25;10(17):3801. 
10 Kindler HL. Understanding the new therapeutic options for mesothelioma. Lancet Oncol. 2021 Oct;22(10): 1353-5.

11 Czarnecka-Kujawa K, de Perrot M, Keshavjee S, Yasufuku K. Endobronchial ultrasound-guided transbronchial needle aspiration mediastinal lymph node staging in malignant pleural mesothelioma. J Thorac Dis. 2019 Feb; 11(2):602-12.

12 Corbin H, Packer CD. False-negative 18F-fluorodeoxyglucose PET/CT in malignant pleural mesothelioma. Cureus. 2021 Aug;13(8):e17263.

13 Piro R, Fontana M, Livrieri F, Menzella F, Casalini E, Taddei S, et al. Pleural mesothelioma: when echo-endoscopy (EUS-B-FNA) leads to diagnosis in a minimally invasive way. Thorac Cancer. 2021 Mar;12(6):981-4.

14 Romei C, Fanni SC, Volpi F, Milazzo A, D'Amore CA, Colligiani L, et al. New updates of the imaging role in diagnosis, staging, and response treatment of malignant pleural mesothelioma. Cancers. 2021 Aug 30;13(17):4377.

15 Tanaka T, Morishita S, Hashimoto M, Nakamichi T, Uchiyama Y, Hasegawa S, et al. Relationship between physical function and health utility in patients undergoing surgical treatment for malignant pleural mesothelioma. Integr Cancer Ther. 2021 Jan-Dec;20:15347354211043508. 